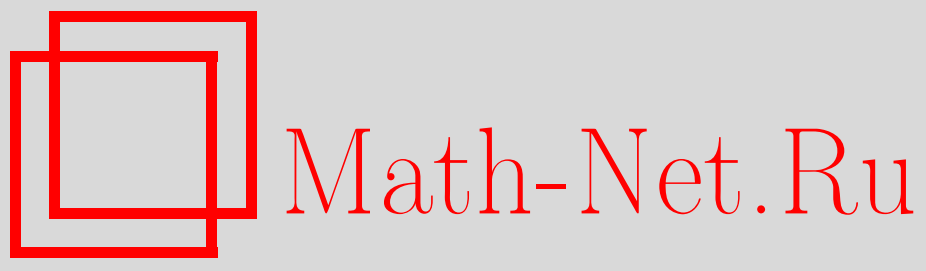

О. Н. Заброда, И. Б. Симоненко, Асимптотическая обратимость и коллективное асимптотическое поведение спектра обобщенных одномерных дискретных сверток, Функи. анализ и его прил., 2004, том 38, выпуск 1, 81-82

DOI: https://doi.org/10.4213/faa98

Использование Общероссийского математического портала MathNet.Ru подразумевает, что вы прочитали и согласны с пользовательским соглашением

http://www . mathnet.ru/rus/agreement

Параметры загрузки:

IP: 54.162 .27 .143

26 апреля 2023 г., 16:31:07

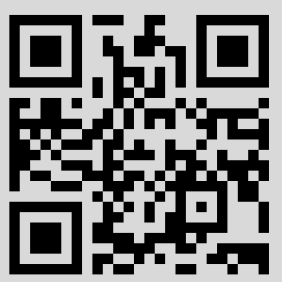




\title{
Асимптотическая обратимость и коллективное асимптотическое поведение спектра обобщенных одномерных дискретных сверток
}

\author{
(c) 2004. О. Н. ЗАБРОДА, И. Б. СИмОНЕНКо
}

Пусть $X \subset \mathbb{R}, x_{0} \in X, \mathfrak{F}$ - множество функций $a: X \times X \times \mathbb{Z} \rightarrow \mathbb{C}$, равномерно непрерывных по совокупности первых двух переменных и удовлетворяющих условию

$$
\sum_{j \in \mathbb{Z}} \sup _{x \in X, y \in X}|a(x, y, j)|<+\infty,
$$

$S=\{z|z \in \mathbb{C}| z \mid,=1\}, s$ - мера-длина на $S$ и $\sigma: \mathfrak{F} \rightarrow C(X \times S)$ - отображение, определяемое равенством

$$
[\sigma(a)](x, t)=\sum_{j \in \mathbb{Z}} a(x, x, j) t^{j} .
$$

Обозначим через $\mathfrak{F}_{0}$ множество, состоящее из всех $a \in \mathfrak{F}$, таких, что замыкание области значений функции $\sigma(a)$ не содержит нуля и $[\sigma(a)]\left(x_{0}, t\right)$ имеет нулевое приращение аргумента по $t \in S$.

Последовательность линейных операторов $A_{n}: \mathbb{C}^{n} \rightarrow \mathbb{C}^{n}$ будем называть асимптотически обратимой, если существуют такие $N \in \mathbb{N}$ и $C>0$, что $A_{n}$ обратим и $\left\|\left(A_{n}\right)^{-1}\right\| \leqslant C$ для каждого натурального $n>N$. При этом пространство $\mathbb{C}^{n}$ мы считаем снабженным евклидовой нормой.

Пусть $E: \mathbb{N} \rightarrow(0,+\infty), E(n) \underset{n \rightarrow+\infty}{\longrightarrow}+\infty$ и выполняется одно из следующих двух условий:

1) $X=[0,1]$ и $E(n)=n$;

2) $X=(0,+\infty)$ и $n / E(n) \underset{n \rightarrow+\infty}{\longrightarrow}+\infty$.

Теорема 1. Пусть $a \in \mathfrak{F}_{0}$. Тогда последовательность операторов $A_{n}(n \in \mathbb{N})$, действующих в пространствах $\mathbb{C}^{n}$ по правилу

$$
\left(A_{n} v\right)(k)=\sum_{j=1}^{n} a\left(\frac{k}{E(n)}, \frac{j}{E(n)}, k-j\right) v(j),
$$

асимптотически обратима.

Tеорема 2. Пусть $a, \delta \in \mathfrak{F}, \delta$ не зависит от первых двух переменных, $\delta(x, y, 0)=1 u \delta(x, y, j)=0$ nри $j \in \mathbb{Z} \backslash\{0\}$. Пусть, далее, $G$ - множество тех $\lambda \in \mathbb{C}$, для которых $а-\lambda \delta \in \mathfrak{F}_{0}, F=\mathbb{C} \backslash G, D$ - открытое подмножество в $\mathbb{C}$, содержащее в себе $F, u A_{n}$ - последовательность операторов, определяемая равенством (1). Тогда существует такое $N \in \mathbb{N}$, что для каждого натурального $n>N$ спектр оператора $A_{n}$ вложен в $D$.

Для линейного оператора $A$, действующего в конечномерном пространстве, и функции $f$ комплексного переменного положим $\Phi_{f}(A)=\sum_{\lambda \in \Lambda} \rho(\lambda) f(\lambda)$, где $\Lambda$ - множество собственных чисел оператора $A$, находящихся в области определения функции $f$, а $\rho(\lambda)$ - кратность собственного числа $\lambda$. 
ТЕОРема 3. Пусть выполнены условия теоремы 2 и $f$-аналитическая функция комплексного переменного, определенная в D. Тогда

$$
\frac{\Phi_{f}\left(A_{n}\right)}{n}-\frac{E(n)}{2 \pi n} \int_{[0, n / E(n)] \times S} \psi(x, t) d x d s \underset{n \rightarrow+\infty}{\longrightarrow} 0,
$$

где $\psi(x, t)=f([\sigma(a)](x, t))$.

Комментарии. Впервые коллективное асимптотическое поведение спектра для самосопряженных теплицевых матриц, т.е. для матриц вида $b_{k, j}=a_{k-j}$ $(k, j \in\{1, \ldots, n\})$, где $a_{k}$ удовлетворяют условию $a_{-k}=\bar{a}_{k}$, исследовал Сегё $[1 ; 2$, гл. 5]. Критерий асимптотической обратимости операторов умножения на теплицевы матрицы (не обязательно самосопряженные) получили Гохберг и Фельдман $[3,4]$. Используя специальную конструкцию асимптотически обратного, Видом [5] существенно усилил результаты Сегё, освободившись от условий самосопряженности. Асимптотическую обратимость и коллективное асимптотическое поведение спектра для операторов вида (1) в случае 1$)(E(n)=n$, $X=[0,1])$, когда функция $a$ не зависит от второго аргумента, изучили Эрхард и Шао [6]. Значительную трудность при этом представляет эффективное построение асимптотически обратного оператора специального вида, удобного для последующего исследования асимптотического поведения спектра. Мы используем несколько иную конструкцию. Она позволяет провести исследование и в случае 2).

\title{
Литература
}

1. Szego G. Medd. Lunds Univ. Mat. Sem. Suppl. Band Festkrift Marcel Riesz, 228238 (1952). 2. Гренандер У., Сегё Г. Теплицевы формы и их приложения. ИЛ, М., 1961. 3. Гохберг И. Ц., Фелъдман И. А. Докл. АН СССР, 165, №2, 268-271 (1965). 4. Гохберг И. Ц., Фелъдман И. А. Уравнения в свертках и проекционные методы их решения. Наука, M., 1971. 5. Widom H. Adv. in Math., 21, 1-29 (1976). 6. Ehrhardt T., Shao B. J. Fourier Analysis Application, 7, No. 1, 71-92 (2001).

Ростовский госуниверситет, механико-математический факультет e-mail: isim@math.rsu.ru

Поступило в редакцию 17 октября 2002 г.

\section{Квазиинвариантные меры и неприводимые представления индуктивного предела специальных линейных групп*}

\author{
(c) 2004. А. В. Косяк
}

1. С каждым действием $\alpha: G \rightarrow \operatorname{Aut}(X)$ группы $G$ на $G$-пространстве $X$ и $G$-квазиинвариантной мерой $\mu$ на $X$ можно связать унитарное представление $\pi^{\alpha, \mu, X}: G \rightarrow U\left(L^{2}(X, d \mu)\right)$ группы $G$ по формуле

$$
\left(\pi_{t}^{\alpha, \mu, X} f\right)(x)=\left(d \mu\left(\alpha_{t^{-1}}(x)\right) / d \mu(x)\right)^{1 / 2} f\left(\alpha_{t^{-1}}(x)\right), \quad f \in L^{2}(X, d \mu) .
$$

*Работа выполнена при частичной поддержке гранта DFG 436 UKR 113/72. 